\title{
openheart Inconsistency in aortic stenosis severity between CT and echocardiography: prevalence and insights into mechanistic differences using computational fluid dynamics
}

\author{
Tarun Kumar Mittal, ${ }^{\oplus 1,2}$ Luise Reichmuth, ${ }^{1}$ Sanjeev Bhattacharyya, ${ }^{\oplus 1}$ Manish Jain, ${ }^{1}$ \\ Aigul Baltabaeva, ${ }^{3}$ Shelley Rahman Haley, ${ }^{1}$ Saeed Mirsadraee, ${ }^{1,2}$ \\ Vasileios Panoulas, ${ }^{2,3}$ Tito Kabir, ${ }^{3}$ Edward David Nicol, ${ }^{1,2}$ Miles Dalby, ${ }^{2,3}$ \\ Quan Long ${ }^{4}$
}

\begin{abstract}
- Additional material is published online only. To view, please visit the journal online (http://dx.doi.org/10.1136/ openhrt-2019-001044)
\end{abstract}

To cite: Mittal TK, Reichmuth L, Bhattacharyya S, et al. Inconsistency in aortic stenosis severity between CT and echocardiography: prevalence and insights into mechanistic differences using computational fluid dynamics. Open Heart 2019;6:e001044. doi:10.1136/ openhrt-2019-001044

Received 6 March 2019 Revised 2 June 2019 Accepted 4 July 2019

\section{Check for updates}

(C) Author(s) (or their employer(s)) 2019. Re-use permitted under CC BY-NC. No commercial re-use. See rights and permissions. Published by BMJ.

${ }^{1}$ Heart Assessment, Royal Brompton and Harefield NHS Foundation Trust, London, , UK ${ }^{2}$ Faculty of Medicine, NHLI, Imperial College London, London, United Kingdom ${ }^{3}$ Cardiology, Royal Brompton and Harefield NHS Foundation Trust, London, United Kingdom ${ }^{4}$ Institute of Bioengineering, Brunel University, Uxbridge, Middlesex, UK

Correspondence to Dr Tarun Kumar Mittal; t.mittal@ outlook.com

\section{ABSTRACT}

Objectives The aims of this study were to evaluate the inconsistency of aortic stenosis (AS) severity between CT aortic valve area (CT-AVA) and echocardiographic Doppler parameters, and to investigate potential underlying mechanisms using computational fluid dynamics (CFD). Methods A total of 450 consecutive eligible patients undergoing transcatheter AV implantation assessment underwent CT cardiac angiography (CTCA) following echocardiography. CT-AVA derived by direct planimetry and echocardiographic parameters were used to assess severity. CFD simulation was performed in 46 CTCA cases to evaluate velocity profiles.

Results A CT-AVA $>1 \mathrm{~cm}^{2}$ was present in $23 \%$ of patients with echocardiographic peak velocity $\geq 4 \mathrm{~m} / \mathrm{s}(\mathrm{r}=-0.33)$ and in $15 \%$ patients with mean Doppler gradient $\geq 40$ $\mathrm{mm} \mathrm{Hg}(\mathrm{r}=-0.39)$. Patients with inconsistent severity grading between CT and echocardiography had higher stroke volume index (43 vs $38 \mathrm{~mL} / \mathrm{m}^{2}, \mathrm{p}<0.003$ ) and left ventricular outflow tract (LVOT) flow rate (235 vs 192 $\mathrm{cm}^{3} / \mathrm{s}, \mathrm{p}<0.001$ ). CFD simulation revealed high flow, either in isolation ( $p=0.01)$, or when associated with a skewed velocity profile $(p=0.007)$, as the main cause for inconsistency between CT and echocardiography. Conclusion Severe AS by Doppler criteria may be associated with a CT-AVA $>1 \mathrm{~cm}^{2}$ in up to a quarter of patients. CFD demonstrates that haemodynamic severity may be exaggerated on Doppler analysis due to high LVOT flow rates, with or without skewed velocity profiles, across the valve orifice. These factors should be considered before making a firm diagnosis of severe AS and evaluation with CT can be helpful.

\section{INTRODUCTION}

Aortic stenosis (AS) is the most common valve disorder in Western countries with an increasing prevalence due to the ageing population. ${ }^{1}$ Transthoracic echocardiography (TTE) is the first-line modality in

\section{Key questions}

What is already known about this subject?

- Inconsistencies between the different echocardiographic parameters for grading aortic stenosis (AS) severity and with catheterisation are already known. Doppler parameters are flow-dependent, increasing with high output states, but the latter are rarely considered in clinical practice. The elliptical shape of left ventricular outflow tract is known to cause underestimation of aortic valve area (AVA) from the continuity equation, and thus overestimate the AS.

What does this study add?

- Almost one-fourth of patients with severe AS on Doppler criteria may have an AVA of $>1 \mathrm{~cm}^{2}$ on direct planimetry using cardiac $\mathrm{CT}$ angiography and are associated with lower valve calcification. This inconsistency results from high flow through the outflow tract possibly causing a skewed velocity profile through the AV orifice.

How might this impact on clinical practice?

- Patients with suspected severe AS on echocardiographic criteria can benefit from assessment of AVA on cardiac CT angiography (besides calcification in the valve) to confirm the diagnosis.

the assessment of AS using haemodynamic Doppler parameters, such as maximum flow velocity $\left(\mathrm{V}_{\max }\right)$ and mean pressure gradient (MG), across the valve along with the aortic valve area (E-AVA) derived from the continuity equation. ${ }^{2-4}$

The aortic valve area (AVA) is theoretically considered to best represent the severity of AS, as it is less affected by flow compared with velocity and gradients. In the 1980s, E-AVA and Doppler parameters were validated against Gorlin's AVA (G-AVA) and haemodynamic 
measurements obtained from cardiac catheterisation $(\mathrm{CC})^{56}$ and outcome data. ${ }^{7}$ However, inconsistency in AS severity with E-AVA became evident early ${ }^{8}$ and continue to be demonstrated in several studies when compared with G-AVA ${ }^{910}$ and pressure gradients. ${ }^{11-13}$ Underestimation of E-AVA is more common and can cause overdiagnosis of AS severity in the presence of non-severe $\mathrm{V}_{\max }$ and $\mathrm{MG},{ }^{14}$ particularly in the presence of low flow, ${ }^{12}$ resulting in a call for potentially abandoning E-AVA as a primary diagnostic parameter for AS diagnosis. ${ }^{15}$ The main reason now considered to cause a smaller E-AVA is the measurement of the smaller dimension of the elliptical left ventricular outflow tract (LVOT) on TTE, resulting in a smaller area using the continuity equation. ${ }^{16}$

CT with its ability to demonstrate and quantify the AV calcium score (AVCS) has been shown to correlate well with both AS severity and prognosis ${ }^{14}$ and has recently been included in the European guidelines for assessment of patients with low-flow, low-gradient stenosis. ${ }^{23}$ Besides, ECG-gated CT cardiac angiography (CTCA) allows measurement of AVA (CT-AVA) by direct planimetry, and unlike AVA obtained from TTE or Gorlin's method, it is not dependent on any assumptions. ${ }^{18}$ Being a three-dimensional (3-D) high-resolution volumetric data, it is routinely possible to align the images parallel to the plane of AV orifice after the scan acquisition, an ability that is unique to CTCA. ${ }^{19}$ Several studies have shown that CT-AVA is slightly larger than E-AVA, ${ }^{18-20}$ with the latter being smaller due to a smaller LVOT size measured on TTE, and the two areas become similar when CT-derived LVOT area is used in the continuity equation. ${ }^{21}$ CT-AVA may, thus, represent a more accurate representation of the anatomical area with less flow dependence.

In patients undergoing CTCA as part of transcatheter AV implantation (TAVI) assessment, we have observed a CT-AVA $>1.0 \mathrm{~cm}^{2}$ even in those with severe TTE haemodynamic parameters, an inconsistency that is less well described. We hypothesised that this might be due to the valve orifice having a heterogeneous velocity profile due to its complex shape, valve thickening and calcification; all factors that may affect the AS severity estimation on TTE. In this study, our aim was to quantify the frequency of Doppler TTE and CT-AVA mismatch in patients with different levels of ventricular function and flow, and explain the cause of this inconsistency using computational fluid dynamics (CFD). CFD is a well-established technique in bioengineering and fluid mechanics that has more recently been used in clinical medicine to assess flow in cardiac and vascular structures. ${ }^{22} 23$ Further details of the CFD technique are given in the online supplementary file.

\section{METHODS}

\section{Study design}

A retrospective analysis was undertaken on consecutive patients diagnosed with severe AS on TTE who underwent ECG-gated cardiac CT as part of TAVI assessment over 5 years (2011-2015) at Harefield Hospital (Royal Brompton and Harefield NHS Foundation Trust), a tertiary cardiothoracic centre. Patients were excluded if they had non-interpretable CT images, bicuspid AV, moderate or greater aortic or mitral regurgitation, prosthetic AV, incomplete CT or TTE data, where the CT and TTE were performed more than 3 months apart, or had intervening balloon valvuloplasty. Patient's demographics and baseline clinical parameters, including body mass index, heart rate, blood pressure, symptoms and cardiovascular risk factors, were collected prospectively. We have attempted to describe the study and report its findings as per the STARD (Standards for Reporting Diagnostic Accuracy) 2015 criteria. $^{24}$

\section{Echocardiography}

All patients underwent comprehensive TTE using commercially available echocardiography machines (Vivid 7 or 9, GE Healthcare, Milwaukee, Wisconsin, USA and IE33, Phillips Healthcare, Andover, Massachusetts, USA) using recommended methods for evaluation and diagnosis of AS. ${ }^{4}$ The left ventricular volumes and ejection fraction (E-LVEF) were measured and calculated using Simpsons' biplane method. LVOT diameter was measured from the parasternal long axis view at early systole. LVOT velocity time integral (VTI) and mean velocity $\left(\mathrm{V}_{\text {mean }}\right)$ were measured using pulse wave Doppler in the apical five-chamber view. The $A V V_{\max }$ and $M G$ were measured using continuous wave Doppler on multiple views. The E-AVA was calculated using the continuity equation. Stroke volume was obtained by multiplying LVOT area by LVOT VTI and was indexed to body surface area (stroke volume index (SVI)). The flow rate was calculated as LVOT area multiplied by LVOT $\mathrm{V}_{\text {mean }}\left(\mathrm{Q}_{\text {mean }}\right)$ or LVOT $\mathrm{V}_{\max }\left(\mathrm{Q}_{\max }\right)$. Velocity ratio was obtained as the ratio of VTI at LVOT and AV. Severe AS on TTE was considered as an E-AVA of $\leq 1 \mathrm{~cm}^{2}, V_{\max } \geq 4 \mathrm{~m} / \mathrm{s}$, or $M G \geq 40 \mathrm{~mm} \mathrm{Hg}^{.}{ }^{34}$

\section{Cardiac CT}

All CT scans were performed on 64-slice (Toshiba Aquilion, Otawara, Japan) or 128-slice (General Electric, Milwaukee, USA) multidetector CT scanners. For AVCS, a standard protocol with $120 \mathrm{kVp}, 3 \mathrm{~mm}$ contiguous acquisition and prospective ECG-gating was used without contrast. CTCA was performed using 100 or $120 \mathrm{kVp}$ and 400-600 mA depending on the patient's body size with retrospective ECG-gating and dose-modulation in the systolic $(0 \%-40 \%)$ phase of the cardiac cycle. Heart rate-lowering drugs were not used due to a diagnosis of severe AS. About $70-90 \mathrm{~mL}$ of non-ionic, low-osmolar contrast (Iopromide, Ultravist 370, Bayer Healthcare, Reading, UK) was administered intravenously. Images were reconstructed with contiguous $2 \mathrm{~mm}$ collimation at every $10 \%$ interval throughout the cardiac cycle. The mean radiation dose was 13.1 and $7.2 \mathrm{mSv}$ (using a conversion factor of 0.014) for CT scans performed on 64-slice and 128-slice scanners, respectively. 


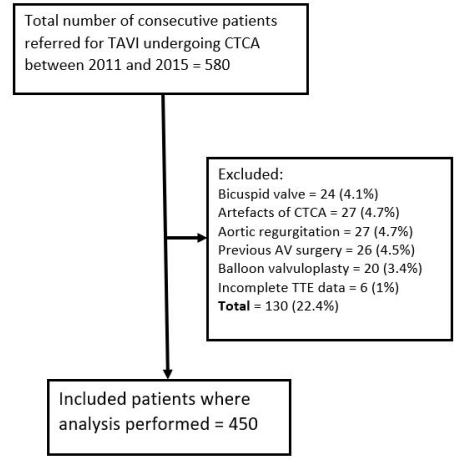

Figure 1 Flow diagram showing recruitment of patients in the study. AV, aortic valve; CTCA, CT cardiac angiogram; TAVI, transcatheter aortic valve replacement; TTE, transthoracic echocardiogram.

\section{CT analysis}

AVCS was quantified using the Agatston method including only the calcification in the AV leaflets. ${ }^{14}$ For calculation of CT-AVA, CTCA data in the systolic phase $(10 \%-30 \%)$ was used. The method used to obtain the $\mathrm{AV}$ orifice in its true short-axis plane in midsystolic phase and to quantify the CT-AVA by direct planimetry is demonstrated in figure 1. A CT-AVA of $\leq 1 \mathrm{~cm}^{2}$ was considered as severe AS. ${ }^{4}$ End-diastolic and end-systolic phases were used to obtain LV volumes, CT-SVI and ejection fraction CT-LVEF. All post-processing was performed by a level 3 SCCT (Society of Cardiovascular Computed Tomography) cardiac radiologist, with $>10$ years' experience, using advanced post-processing tools (Vital Images, Minnetonka, Minnesota, USA). All CT evaluation was performed without the knowledge of details of the patient's clinical status and TTE results.

\section{CFD method and analysis}

CTCA image data from 46 patients with a tricuspid valve and $\mathrm{V}_{\max } \geq 4 \mathrm{~m} / \mathrm{s}$ or $\mathrm{MG} \geq 40 \mathrm{~mm} \mathrm{Hg}$ were selected for CFD analysis. Half of these patients had a CT-AVA of $\leq 1 \mathrm{~cm}^{2}$ (consistent grading), while the other half had a CT-AVA of $>1 \mathrm{~cm}^{2}$ (inconsistent grading). The CTCA data at midsystolic phase were uploaded on standard CFD software (ANSYS16.2-CFX, ANSYS, Cannonsburg, Pennsylvania, USA) to generate the 3-D computational mesh and CFD simulation through the LVOT, AV and the aortic root extending into the ascending aorta. Further details of the CFD procedure are given in the online supplementary file.

Both LVOT and AV area were calculated on CFD software. The flow rate at the LVOT was derived from TTE $\mathrm{V}_{\max }$ and CFD-LVOT area, and this was used as the CFD boundary condition at the inlet plane. The distribution of peak velocities (CFD-AV) at the AV orifice was evaluated, and a ratio of $\geq 1.2$ between peak and $V_{\text {mean }}$ across the AV orifice was considered as skewed. Peak pressure gradient (PG) was obtained at the AV orifice. Additionally, the velocity ratio between LVOT and AV were obtained.

\section{Statistical analysis}

Patients were divided into three groups based on echocardiography LV function and LVOT flow: Group A: E-LVEF $\geq 50 \%$ and $\mathrm{SVI} \geq 35 \mathrm{~mL} / \mathrm{m}^{2}$; Group B: E-LVEF $\geq 50 \%$ and SVI $<35 \mathrm{~mL} / \mathrm{m}^{2}$; and Group C: E-LVEF $<50 \%$. The strength of association of severity of AV stenosis on CT-AVA was compared with TTE parameters on a continuous scale within each group using Pearson correlation. Comparison was made across the three groups for the severity of stenosis and the degree of inconsistency was compared using the $\chi^{2}$ test. Continuous variables are presented as mean $\pm \mathrm{SD}$ or median (IQR), while categorical variables are presented as frequencies with percentages.

Patients with severe Doppler parameters $\left(\mathrm{V}_{\max } \geq 4\right.$ $\mathrm{m} / \mathrm{s}$ or $\mathrm{MG} \geq 40 \mathrm{~mm} \mathrm{Hg}$ ) were divided into those with CT-AVA $\leq 1 \quad \mathrm{~cm}^{2}$ (consistent grading) and CT-AVA $>1$ $\mathrm{cm}^{2}$ (inconsistent grading). Imaging parameters were compared between the groups using $\chi^{2}$ test (or Fisher's exact test for CFD parameters) for categorical variables and unpaired t-test or the Mann-Whitney test for continuous variables. Subsequently, multiple logistic regression analysis was used to examine parameters associated with inconsistent grading using a backward selection procedure. Interobserver and intraobserver variability, as well as inter-rater reliability, was performed for measurement of CT-AVA between two SCCT level 3 cardiac CT experts, with $>10$ years of experience, using interclass correlation (ICC) on a random selection of 50 cases with severe Doppler parameters. All analysis was performed using STATA V.13.0. A two-tailed $p$ value of $<0.05$ was considered statistically significant.

\section{RESULTS}

A total of 580 patients referred for TAVI underwent both echo and CT (mean interval $34 \pm 16$ days), of whom $450(84 \%)$ fulfilled the inclusion criteria (figure 1). The baseline clinical characteristics are given in table 1 .

\section{Comparison between CT and TTE}

The majority of patients $(95 \%)$ referred for TAVI had a diagnosis of severe AS due to E-AVA $\leq 1 \mathrm{~cm}^{2}$, while only two-thirds had haemodynamically severe AS (table 2). This could be due to almost one-third of patients been referred for TAVI mainly on the basis of E-AVA $\leq 1 \mathrm{~cm}^{2}$. A CT-AVA $\leq 1 \mathrm{~cm}^{2}$ was also present in $65 \%$ of patients. In Group A patients, the estimation of severe E-AVA was not consistent with CT-AVA in almost one-third of patients $(31 \%)$, with a mean area difference of $28 \%$ (95\% CI $-31 \%$ to $-26 \%$ ). A CT-AVA $>1 \mathrm{~cm}^{2}$ was present in $28 \%$ of patients with $\mathrm{E}-\mathrm{V}_{\max } \geq 4 \mathrm{~m} / \mathrm{s}$, and in $20 \%$ of patients with $\mathrm{E}-\mathrm{MG} \geq 40 \mathrm{~mm} \mathrm{Hg}$. These differences remained in Group B and C patients, with no statistical difference between the groups (table 2).

In the $292 / 450$ patients $\left(65 \%\right.$ ) with a $\mathrm{V}_{\max } \geq 4 \mathrm{~m} / \mathrm{s}$ and/ or $\mathrm{MG} \geq 40 \mathrm{~mm} \mathrm{Hg}, 67$ (23\%) had inconsistent grading $\left(\right.$ CT-AVA $\left.>1 \mathrm{~cm}^{2}\right)$. The univariate analysis between the 


\begin{tabular}{lll}
\hline Table 1 & Baseline clinical characteristics $(\mathrm{n}=450$ patients $)$ \\
\hline Characteristics & Number & Percentage \\
\hline Age in years, mean \pm SD & $81.5 \pm 8.0$ & NA \\
\hline Male sex & 235 & 52.2 \\
\hline Symptomatic & 276 & 63.5 \\
\hline BMI, $\mathrm{kg} / \mathrm{m}^{2}$ & $27.2 \pm 5.3$ & $\mathrm{NA}$ \\
\hline $\mathrm{BSA}, \mathrm{m}^{2}$ & $1.76 \pm 0.16$ & $\mathrm{NA}$ \\
\hline Hypertension & 225 & 50.3 \\
\hline Diabetes & 109 & 24.6 \\
\hline Dyslipidaemia & 186 & 42 \\
\hline Smoking & 49 & 11 \\
\hline Known CAD & 145 & 32.2 \\
\hline
\end{tabular}

$\mathrm{BMI}$, body mass index; BSA, body surface area; CAD, coronary artery disease.

consistent and inconsistent groups is demonstrated in table 3. On logistic regression analysis, lower AV calcification, higher CT-derived stroke volume and higher LVOT flow rates were statistically significant predictors of inconsistency grading with ORs of 0.44 (95\% CI 0.33 to 0.60$), 1.85$ (95\% CI 1.31 to 2.61 ) and 1.76 (95\% CI 1.30 to 2.39$)$, respectively, $(\mathrm{p}<0.05)$.

The ICC for intraobserver and interobserver variability was 0.87 (95\% CI 0.78 to 0.92 ) and $0.78(95 \%$ CI 0.67 to 0.88 ), respectively. The inter-rater reliability among the two experts was $92 \%$.

\section{CFD analysis}

The CFD simulations demonstrated that the inconsistent group had a significantly larger AVA, lower $\mathrm{V}_{\max }$, lower peak PG and a higher velocity ratio $(p<0.001)$ (table 4). On logistic regression analysis, the LVOT flow rate, both absolute and $\geq 450 \mathrm{~cm} / \mathrm{s}^{3}(\mathrm{p}=0.01)$, was found to be significantly greater in the inconsistent group and perhaps the main cause for the high velocity or gradient despite a CT-AVA $>1 \mathrm{~cm}^{2}$. Although there was no significant difference in the velocity profile (CFD$\mathrm{AV}$ ) between the two groups, when combined with a high LVOT flow rate $\geq 450 \mathrm{~cm} / \mathrm{s}^{3}$, a skewed velocity profile (a ratio of $\geq 1.2$ between peak and $\mathrm{V}_{\text {mean }}$ across the AV orifice) was seen to be present in $61 \%$ of inconsistent group patients compared with $22 \%$ of consistent group ( $\mathrm{p}=0.007$ ) (table 4 and figure 2). Although the velocity ratio was significantly different in the two groups $(p<0.001)$, it was $\leq 0.20$ in both, thus limiting its ability to distinguish between them. Different representative examples of CT-AVA and CFD correlation are shown in figure 3.

\section{DISCUSSION}

Our study demonstrates that a CT-derived AVA of $>1 \mathrm{~cm}^{2}$ is present in up to a quarter of patients with severe haemodynamic AS on echocardiography, regardless of ejection fraction and stroke volume. Higher stroke volume and

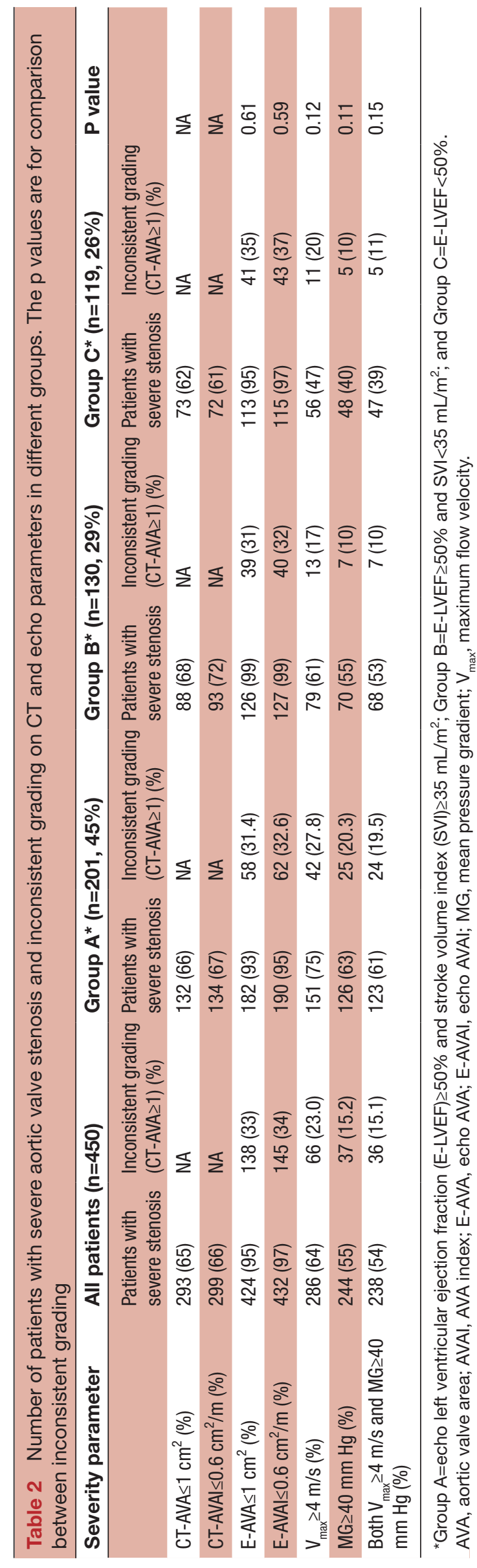




\begin{tabular}{|c|c|c|c|c|}
\hline Imaging criteria & All patients & Consistent grading & Inconsistent grading & $P$ value \\
\hline Number of patients (\%) & $292(65)$ & $225(77)$ & $67(23)$ & \\
\hline Age, years $\pm S D$ & $81.3 \pm 8.4$ & $82.9 \pm 6.8$ & $75.9 \pm 10.8$ & $<0.001$ \\
\hline AVCS, median (IQR) & 3499 (2383-4661) & $3855(2800-4940)$ & $2141(1664-3000)$ & $<0.001$ \\
\hline CT-AVA, mean $\mathrm{cm}^{2}( \pm \mathrm{SD})$ & $0.90(0.3)$ & $0.76(0.14)$ & $1.32(0.25)$ & $<0.001$ \\
\hline E-AVA, mean $\mathrm{cm}^{2}( \pm \mathrm{SD})$ & $0.65(0.2)$ & $0.60(0.2)$ & $0.79(0.16)$ & $<0.001$ \\
\hline $\mathrm{V}_{\max }$, mean m/s (IQR) & $4.5(4.2-5.0)$ & $4.6(4.2-5.0)$ & $4.2(4.2-5.0)$ & $<0.001$ \\
\hline $\mathrm{MG}$, mean $\mathrm{mm} \mathrm{Hg}( \pm \mathrm{SD})$ & $48(42-61)$ & $52(44-62)$ & $40(37-51)$ & $<0.001$ \\
\hline Velocity ratio $( \pm S D)$ & $0.21(0.07)$ & $0.19(0.06)$ & $0.25(0.07)$ & $<0.001$ \\
\hline E-LVEF, \% ( $( \pm S D)$ & $58.8(12.3)$ & $58.6(12.2)$ & $59.7(13.7)$ & 0.51 \\
\hline CT-LVEF, \% ( $( \pm \mathrm{SD})$ & $62.0(15.7)$ & $60.9(15.4)$ & $65.7(13.7)$ & 0.03 \\
\hline $\mathrm{E}-\mathrm{SVI}( \pm \mathrm{SD})$ & $38.9(12.5)$ & $37.6(11.7)$ & $43.6(14.3)$ & $<0.001$ \\
\hline CT-SVI ( $( \pm \mathrm{SD})$ & $41.2(10.8)$ & $39.6(10.2)$ & $46.8(11.1)$ & $<0.001$ \\
\hline LVOT $Q_{\text {mean }}, \mathrm{cm}^{3} / \mathrm{s}( \pm \mathrm{SD})$ & $203(62)$ & $193(55)$ & $238(70)$ & $<0.001$ \\
\hline
\end{tabular}

AVA, aortic valve area; AVCS, aortic valve calcium score; E-AVA, echo AVA; E-LVEF, echo left ventricular ejection fraction;E-SVI, echo stroke volume index; LVOT, left ventricular outflow tract; $M G$, mean pressure gradient; $Q_{\text {mean }}$, mean flow rate; $V_{\text {max }}$, maximum flow velocity.

outflow-tract flow rates on echocardiography are associated with increased inconsistency with CT grading. CFD analysis also supports that high LVOT flow rates are associated with inconsistency and may be responsible for the skewed velocity profile through the AV orifice, thus leading to higher haemodynamic parameters in the presence of larger CT valve area.

As expected, the E-AVA was the most inconsistent parameter when compared with Doppler indices, as well as CT-AVA, and results from the measurement of small LVOT diameter as discussed earlier. ${ }^{151621}$ Another reason that has been used to explain a smaller E-AVA in relation to directly measured AVA is the concept that the former represents the area of the smaller vena contracta distal to the anatomical AVA. ${ }^{25}$ While this may be the case in truly severe AS, it may not be so in mild-to-moderate AS. Using
Gorlin's equation, it has been demonstrated that an MG of $40 \mathrm{~mm} \mathrm{Hg}$ and $\mathrm{V}_{\max }$ of $4 \mathrm{~m} / \mathrm{s}$ better corresponds to E-AVA of $0.75 \mathrm{~cm}^{2}$ and $0.82 \mathrm{~cm}^{2}$, respectively, thus questioning the $1.0 \mathrm{~cm}^{2}$ cut-off value on TTE, which was based on Gorlin's equation. ${ }^{11}$

We did not attempt to estimate corrected E-AVA based on CT-derived LVOT areas as this was not the primary objective of this study. The overestimation of AS severity based on E-AVA cut-off of $1.0 \mathrm{~cm}^{2}$ may explain some asymptomatic patients or those with low-flow labelled as severe AS.

It is well known that velocities and gradients are flow-dependent, but clinically more careful consideration is given to low flow in the context of AS assessment rather than high flow. A few studies have evaluated the role of high flow on AS parameters in the context of exercise,

Table 4 Comparison of different parameters in computational flow dynamics (CFD) patients with and without CT-AVA $\leq 1$

\begin{tabular}{|c|c|c|c|}
\hline Parameter & $\begin{array}{l}\text { Consistent grading } \\
(n=23)\end{array}$ & Inconsistent grading $(\mathrm{n}=23)$ & $P$ value \\
\hline AVCS, median (IQR) & $3381(2843-5000)$ & 2004 (1710-2793) & 0.001 \\
\hline CFD-AVA, $\mathrm{cm}^{2}, \pm \mathrm{SD}$ & $0.7 \pm 0.2$ & $1.2 \pm 0.2$ & $<0.001$ \\
\hline $\mathrm{CFD}-\mathrm{V}_{\max } \pm \mathrm{SD}(\mathrm{m} / \mathrm{s})$ & $7.2 \pm 2.3$ & $5.7 \pm 1.3$ & 0.01 \\
\hline $\mathrm{CFD}$ peak $\mathrm{PG}, \mathrm{mm} \mathrm{Hg}, \pm \mathrm{SD}$ & $206 \pm 136$ & $106 \pm 54$ & 0.002 \\
\hline CFD LVOT/AV velocity ratio (95\% Cl) & $0.11(0.09$ to 0.17$)$ & $0.20(0.16$ to 0.23$)$ & $<0.001$ \\
\hline CFD-AV orifice velocity profile ratio, median (IQR) & $1.26(1.16-1.45)$ & $1.24(1.17-1.37)$ & 0.63 \\
\hline CFD-AV orifice velocity profile ratio $\geq 1.2(\%)$ & $14(61)$ & $16(70)$ & 0.53 \\
\hline LVOT $Q_{\text {max }}, \mathrm{cm}^{3} / \mathrm{s}$, mean $\pm S D$ & $414 \pm 103$ & $552 \pm 157$ & 0.001 \\
\hline LVOT $Q_{\max } \geq 450(\%)$ & $8(35)$ & $19(83)$ & 0.01 \\
\hline Both CFD-AV orifice velocity ratio $\geq 1.2$ and LVOT Flow rate $\geq 450$ (\%) & $5(22)$ & $14(61)$ & 0.007 \\
\hline
\end{tabular}

$A V$, arotic valve; AVA, AV area; AVCS, AV calcium score; LVOT, left ventricular outflow tract; $P G$, pressure gradient; $Q_{\text {max }}$, maximum flow rate; $\mathrm{V}_{\text {max }}$, maximum flow velocity. 

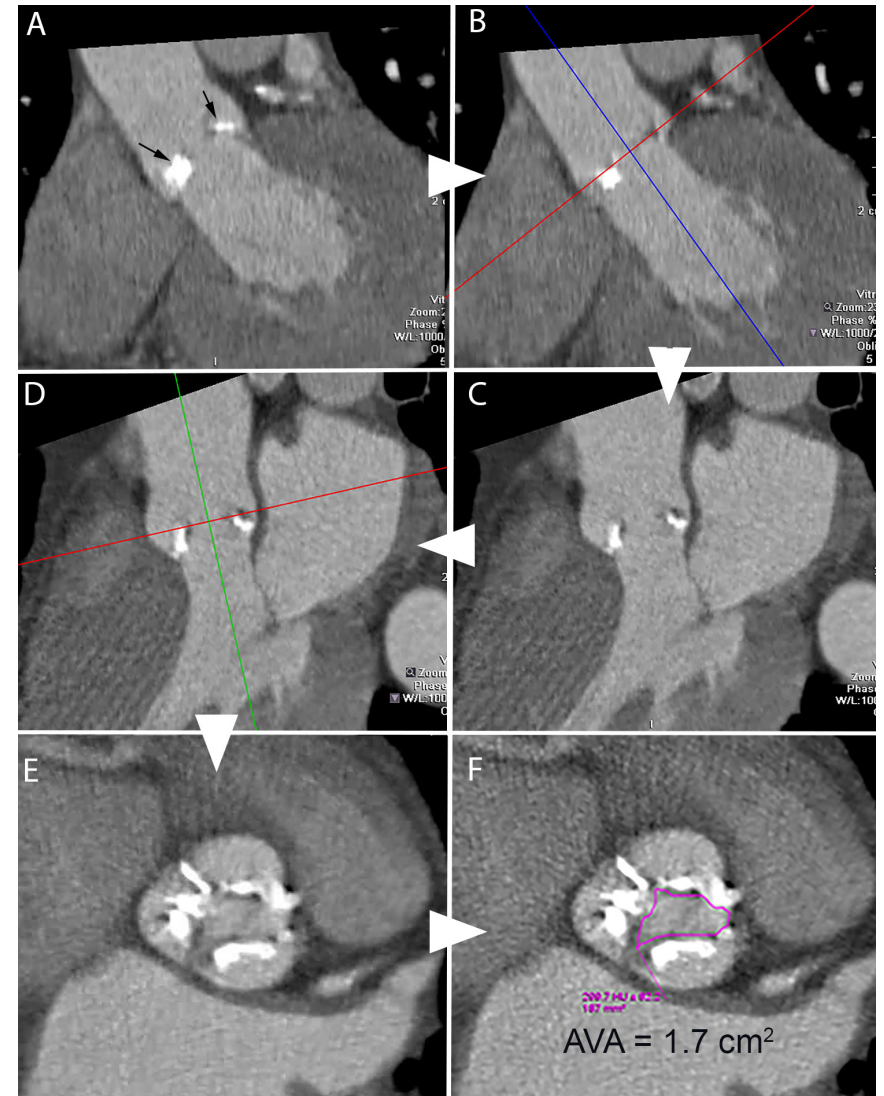

Figure 2 Measurement of CT aortic valve (AV) area. CT cardiac angiography images demonstrating a method of obtaining $A V$ area in a patient with tricuspid valve with calcification. Midsystolic phase of the cardiac cycle with the maximum opening of the AV is first selected. Starting from a coronal image (A) with non-coronary (left) and left leaflets (right) (black arrows), one of the crosshair is aligned (B) such that it passes parallel to the AV plane and the other perpendicular through the AV orifice, resulting in a left ventricular outflow tract view (C). The crosshair is further aligned parallel to the valve plane (D) to produce a true short-axis view through the AV (E). By scrolling in this plane, the smallest orifice is obtained and direct planimetry of the orifice $(F)$ is performed along the inner edges of the leaflets to obtain the AV area.

resulting in an increase of $\mathrm{V}_{\max }$ and $\mathrm{MG}^{26}$ However, increased haemodynamic parameters have been considered to represent severe AS irrespective of the valve area, although caution has been suggested in those with a high physiological cardiac output state. ${ }^{2}$ It is only in patients with bicuspid and prosthetic AV where eccentric jets have been demonstrated to cause greater pressure loss as the jet collides with the aortic wall resulting in more energy loss due to heat, flow separation and vortex formation leading to increased pressure gradients on both TTE and cardiac catheterisation studies. ${ }^{27} 28$ There is a lack of studies demonstrating a skewed velocity profile through the native AV orifice as a potential cause of increased measured Doppler velocities. An in-vitro study revealed skewed flow through stenotic bioprosthetic valves and recommended multiple views for velocity

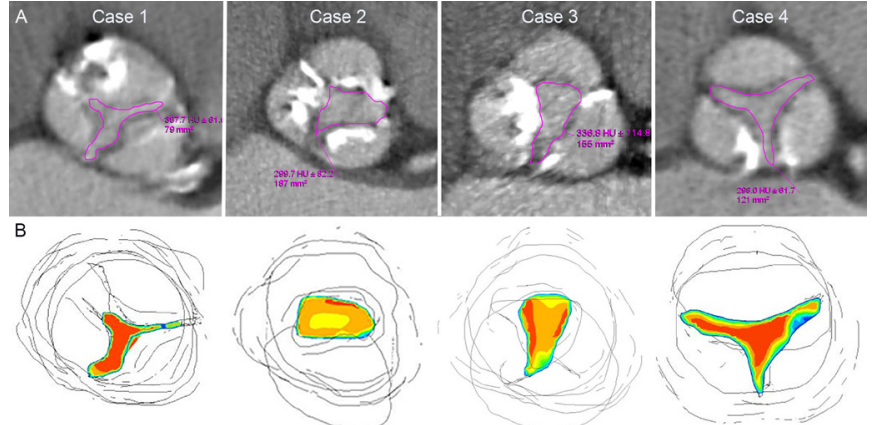

Figure 3 Cases demonstrating the correlation of CT aortic valve area (CT-AVA) with computational flow dynamics. All cases have echocardiographic $V_{\max }>4 \mathrm{~m} / \mathrm{s}$ and mean gradient $>40 \mathrm{~mm}$ Hg. (A) Upper row demonstrates CT-AVA by direct planimetry, while lower row $(B)$ depicts computational fluid dynamics simulation (red colour representing highest velocity, blue intermediate and yellow lowest). Case 1 represents consistent aortic stenosis grading with CT$\mathrm{AVA}=0.8 \mathrm{~cm}^{2}$, flow rate $=517$ and flat velocity profile of 1.1 . Cases 2, 3 and 4 represent inconsistent grading with CTAVA $=1.7 \mathrm{~cm}^{2}, 1.5 \mathrm{~cm}^{2}$ and $1.2 \mathrm{~cm}^{2}$; high flow rate of 604,701 and 530; and skewed velocity profile of 1.3, 1.24 and 1.3, respectively.

estimation. ${ }^{25}$ A recent 3-D cardiovascular MR flow analysis has also revealed a heterogeneous profile through the vena contracta and demonstrated that the simplified Bernoulli equation can overestimate MG by an average of $54 \% .^{29}$ The Bernoulli equation, which is based on the Navier-Stokes equation, relies on certain assumptions including a uniform velocity profile through the orifice. As we found that a skewed velocity profile is significant when associated with high LVOT flow rate rather than on its own, it is possible that the skewed profile is caused by the high flow. CTCA allows more accurate delineation of valve morphology and opening, and along with the use of CFD techniques, this variation in the velocity across the valve orifice is easier to determine.

The orifice morphology and flow channel of diseased $\mathrm{AV}$ are complex and not conducive to uniform flow profiles. Edges of calcified leaflets and variable commissure anatomy may cause local flow disturbance and contribute to a skewed orifice velocity profile, particularly in combination with high LVOT flow rates. The highest velocity on continuous Doppler used to calculate AS severity may not represent a true picture for the whole AV orifice and may, therefore, overestimate the degree of stenosis.

The phenomenon of pressure recovery in the ascending aorta has been shown to cause greater pressure gradients across the AV on TTE when compared with cardiac catheterisation. ${ }^{3}$ The magnitude of $\mathrm{PR}$ is considered small in adults, occurring mainly if the aorta is $<3 \mathrm{~cm}$ in diameter. Falsely high gradients can also be caused by moderate or more aortic regurgitation or subvalvular obstruction. ${ }^{3}$ We had excluded these patients in our study.

ECG-gated CTCA through the systolic phase of the cardiac cycle allows assessment of valve morphology and 
opening, irrespective of the degree of calcification. ${ }^{18}$ Measurement of CT-AVA requires the midsystolic phase with the maximum valve opening and precise orthogonal alignment in short-axis of the valve plane to calculate the minimum orifice area. Unlike E-AVA or Doppler criteria, CT-AVA is not based on assumptions and is less flow-dependent. Calcification does not appear to hinder planimetry of CT-AVA, as it seems to deposit within the cusp margins. ${ }^{3}$ The degree of AV calcification, a parameter that is now well accepted as a marker for AS severity, ${ }^{3}{ }^{14}$ was significantly lower in patients with inconsistent AS in this study.

In ECG-gated studies that include end-systolic and end-diastolic phases, an accurate estimation of LV function can be made by allowing accurate estimation of stroke volume and flow. Both CT and MRI angiographic studies have shown that this results in improved classification of AS based on flow and function. ${ }^{30}$

\section{Study limitations}

TTE and CT studies were not performed simultaneously which can potentially result in differences in haemodynamic-loading conditions and variation in measured parameters. However, our data reveal a consistent pattern of inconsistency across all three groups. As this is a retrospective analysis of clinically performed studies (TTE and CT), there may be inaccuracies in the parameters obtained; however, the severe AS based on the TTE drove the clinical referral for TAVI and hence, a CT was performed. We did not evaluate the role of cusp shape, which could play a role in forming a skewed velocity profile. Echocardiographic parameters of AS severity have been widely used clinically and validated in follow-up outcome studies, although the value of these parameters for mortality has been questioned in a recent large study. ${ }^{31}$ Similar outcome studies would be required for CT-AVA in patients with different ventricular function for the technique to be accepted.

\section{CONCLUSION}

Our study demonstrates that almost a quarter of patients with severe AS by TTE Doppler criteria demonstrate a CT-AVA $>1 \mathrm{~cm}^{2}$. CTCA is better able to demonstrate the complex morphology of the AV orifice and cusp calcification. The inconsistency between echo and CT appears to be due to high flow rates alone or by latter causing a skewed velocity profile across the valve orifice. The peak velocity obtained on Doppler may not represent an accurate value for the whole orifice leading to a systematic 'overestimation' of stenosis severity. In patients with suspected severe AV stenosis on echo, further evaluation of CT-AVA along with valve calcification is recommended to confirm the stenosis severity before any valve intervention is undertaken.

Acknowledgements Authors are grateful for the statistical assistance provided by Paul Bassett.
Contributors TKM developed the hypothesis and design the study. LR, SB, MJ and TKM collected the clinical, echocardiographic and CT data. SM was involved in the study of interobserver variability. QL was responsible for the computational fluid dynamics. TM, SB, AB, SRH, VP, TK, EDN, MD and QL assisted in study drafts and final version.

Funding This research has received no specific grant from any funding agency in the public, commercial or not-for-profit sectors.

Competing interests No, there are no competing interests for any author. Patient consent for publication Not required.

Ethics approval NHS (National Health Service) research ethics approval was obtained (IRAS Project ID: 2014HS002H).

Provenance and peer review Not commissioned; externally peer reviewed. Data availability statement Data are available on reasonable request.

Open access This is an open access article distributed in accordance with the Creative Commons Attribution Non Commercial (CC BY-NC 4.0) license, which permits others to distribute, remix, adapt, build upon this work non-commercially, and license their derivative works on different terms, provided the original work is properly cited, appropriate credit is given, any changes made indicated, and the use is non-commercial. See: http://creativecommons.org/licenses/by-nc/4.0/.

\section{REFERENCES}

1. Otto CM, Prendergast B. Aortic-Valve stenosis - from patients at risk to severe valve obstruction. N Engl J Med 2014;371:744-56.

2. Baumgartner $\mathrm{H}$, Falk $\mathrm{V}$, Bax JJ, et al. ESC/EACTS guidelines for the management of valvular heart disease. Eur Heart $J$ 2017;2017:2739-91.

3. Baumgartner $\mathrm{H}$, Hung J, Bermejo J, et al. Recommendations on the echocardiographic assessment of aortic valve stenosis: a focused update from the European association of cardiovascular imaging and the American Society of echocardiography. Eur Heart J Cardiovasc Imaging 2017;18:254-75.

4. Nishimura RA, Otto CM, Bonow RO, et al. 2014 AHA/ACC guideline for the management of patients with valvular heart disease: a report of the American College of Cardiology/American heart association Task force on practice guidelines. Circulation 2014;129:e521-643.

5. Currie PJ, Seward JB, Reeder GS, et al. Continuous-Wave Doppler echocardiographic assessment of severity of calcific aortic stenosis: a simultaneous Doppler-catheter correlative study in 100 adult patients. Circulation 1985;71:1162-9.

6. Oh JK, Taliercio CP, Holmes DR, et al. Prediction of the severity of aortic stenosis by Doppler aortic valve area determination: prospective Doppler-catheterization correlation in 100 patients. $J \mathrm{Am}$ Coll Cardiol 1988;11:1227-34.

7. Otto CM, Burwash IG, Legget ME, et al. Prospective study of asymptomatic valvular aortic stenosis. Clinical, echocardiographic, and exercise predictors of outcome. Circulation 1997;95:2262-70.

8. Danielsen R, Nordrehaug JE, Vik-Mo H. Factors affecting Doppler echocardiographic valve area assessment in aortic stenosis. Am J Cardiol 1989;63:1107-11.

9. Yang C-S, Marshall ES, Fanari Z, et al. Discrepancies between direct catheter and echocardiography-based values in aortic stenosis. Cathet Cardiovasc Interv 2016;87:488-97.

10. Fischer JL, Haberer T, Dickson D, et al. Comparison of Doppler echocardiographic methods with heart catheterisation in assessing aortic valve area in 100 patients with aortic stenosis. $\mathrm{Br}$ Heart $J$ 1995;73:293-8.

11. Minners J, Allgeier M, Gohlke-Baerwolf C, et al. Inconsistencies of echocardiographic criteria for the grading of aortic valve stenosis. Eur Heart J 2008;29:1043-8.

12. Minners J, Allgeier M, Gohlke-Baerwolf C, et al. Inconsistent grading of aortic valve stenosis by current guidelines: haemodynamic studies in patients with apparently normal left ventricular function. Heart 2010;96:1463-8.

13. Nies R, Pfister R, Kuhr K, et al. Inconsistency in hemodynamic characterization of severe aortic stenosis. Int $\mathrm{J}$ Cardiol 2015;197:309-11.

14. Clavel M-A, Messika-Zeitoun D, Pibarot P, et al. The complex nature of discordant severe calcified aortic valve disease grading: new insights from combined Doppler echocardiographic and computed tomographic study. J Am Coll Cardiol 2013;62:2329-38.

15. Baumgartner $\mathrm{H}$. Should we forget about valve area when assessing aortic stenosis? Heart 2019;105:92-3.

16. Doddamani S, Bello R, Friedman MA, et al. Demonstration of left ventricular outflow tract eccentricity by real time 3D 
echocardiography: implications for the determination of aortic valve area. Echocardiography 2007;24:860-6.

17. Clavel M-A, Pibarot P, Messika-Zeitoun D, et al. Impact of aortic valve calcification, as measured by MDCT, on survival in patients with aortic stenosis: results of an international registry study. J Am Coll Cardiol 2014;64:1202-13.

18. Lembcke A, Woinke M, Borges AC, et al. Grading of aortic valve stenosis at 64-slice spiral computed tomography: comparison with transthoracic echocardiography and calibration against cardiac catheterization. Invest Radiol 2009;44:360-8.

19. Larsen LH, Kofoed KF, Carstensen HG, et al. Aortic valve area assessed with 320-detector computed tomography: comparison with transthoracic echocardiography. Int J Cardiovasc Imaging 2014;30:165-73.

20. Anger T, Bauer V, Plachtzik C, et al. Non-Invasive and invasive evaluation of aortic valve area in 100 patients with severe aortic valve stenosis: comparison of cardiac computed tomography with echo (transesophageal/transthoracic) and catheter examination. $J$ Cardiol 2014;63:189-97.

21. Halpern EJ, Mallya R, Sewell M, et al. Differences in aortic valve area measured with CT planimetry and echocardiography (continuity equation) are related to divergent estimates of left ventricular outflow tract area. AJR Am J Roentgenol 2009;192:1668-73.

22. Wong KKL, Wang D, Ko JKL, et al. Computational medical imaging and hemodynamics framework for functional analysis and assessment of cardiovascular structures. Biomed Eng Online 2017;16:35.
23. Morris PD, Narracott A, von Tengg-Kobligk H, et al. Computational fluid dynamics modelling in cardiovascular medicine. Heart 2016;102:18-28.

24. Bossuyt PM, Reitsma JB, Bruns DE, et al. STARD 2015: an updated list of essential items for reporting diagnostic accuracy studies. BMJ 2015;351:h5527.

25. Yoganathan AP. Fluid mechanics of aortic stenosis. Eur Heart $J$ 1988;9(Suppl E):13-17.

26. Lancellotti P, Lebois F, Simon M, et al. Prognostic importance of quantitative exercise Doppler echocardiography in asymptomatic valvular aortic stenosis. Circulation 2005;112(9 Suppl):I377-I382.

27. Donal E, Novaro GM, Deserrano D, et al. Planimetric assessment of anatomic valve area overestimates effective orifice area in bicuspid aortic stenosis. J Am Soc Echocardiogr 2005;18:1392-8.

28. Abbas AE, Franey LM, Lester $\mathrm{S}$, et al. The role of jet eccentricity in generating disproportionately elevated transaortic pressure gradients in patients with aortic stenosis. Echocardiography 2015:32:372-82.

29. Donati F, Myerson S, Bissell MM, et al. Beyond Bernoulli: improving the accuracy and precision of noninvasive estimation of peak pressure drops. Circ Cardiovasc Imaging 2017;10:e005207.

30. Stähli BE, Stadler T, Holy EW, et al. Impact of stroke volume assessment by integrating multi-detector computed tomography and Doppler data on the classification of aortic stenosis. Int J Cardiol 2017;246:80-6.

31. Capoulade R, Le Ven F, Clavel M-A, et al. Echocardiographic predictors of outcomes in adults with aortic stenosis. Heart 2016;102:934-42. 